\title{
Multiple risk prediction of myocardial infarction in women as compared with men
}

\author{
L. WILHELMSEN, C. BENGTSSON, D. ELMFELDT, A. VEDIN, \\ C. WILHELMSSON, G. TIBBLIN, O. LINDQVIST, AND H. WEDEL
}

From the Section of Preventive Cardiology, Department of Medicine I, Sahlgrenska Hospital, S-413 45 Göteborg, Sweden

A study was undertaken to determine whether women who suffer a myocardial infarction in spite of the low frequency in this sex, are more heavily burdened by coronary risk factors than other women and men of similar age. Data were obtained from two random population samples of 855 men and 396 women aged 50 years and compared with all men $(n=160)$ and women $(n=60)$ suffering non-fatal primary myocardial infarcts during 1968 to 1970 and 1968 to 1974, respectively.

Women with infarction had higher blood pressure values, higher triglyceride values, and smoked considerably more than women in the general population. The cholesterol values were not significantly higher in the women with infarction.

The multivariate risk, based on cholesterol level, systolic blood pressure, and smoking habits, in the women with infarction was very similar to that in the men with myocardial infarction. There was no major difference in risk between pre- and postmenopausal women. A higher prevalence of diabetes mellitus in the women with infarction compared with the general population could not explain their increased risk for myocardial infarction. Thus, other factors must be operating in addition to the traditional risk factors.

The incidence of myocardial infarction is higher in men than in women. From a Myocardial Infarction Register, we found the incidence to be about 6 times higher in men than in women in the age group 45 to 54 years (Elmfeldt et al., 1975a). The prevalence of angina pectoris as determined by a questionnaire was, however, similar in the two sexes (Bengtsson, 1969). Because of the low incidence of myocardial infarction in women, few population studies have been able to give an indication of prospective risk factors for this disease in women. In most communities, the only way of finding risk factors in women would be to perform well-designed case control studies.

In the Framingham study (Kannel and Castelli, 1972), it was found that the same risk factors seem to operate in women as in men. It has been found that the mean age at the menopause was somewhat lower among women with myocardial infarction than among women in general population samples of similar age (Bengtsson, 1973; Kannel et al., 1975). In a report on myocardial infarction women several of the patients were still menstruating (Bengtsson, 1973), suggesting the protective effect Received for publication 14 April 1977 of the female hormone pattern can be nullified. A question of relevance in this connection is whether those women who suffer a myocardial infarct, but who are still menstruating, are more heavily burdened by coronary risk factors than other women and men of similar age.

The present paper reports some data concerning multivariate risk prediction based on serum cholesterol level, systolic blood pressure, and smoking habits, with the aim of comparing the risk factor profile in the male and female population and in men and women who had suffered a myocardial infarct. Prospective data from a population study in men were available (Wilhelmsen et al., 1973).

\section{Study population and methods}

Since 1 January 1968, all cases of acute myocardial infarction occurring in the population of Göteborg in certain age groups have been registered by a special organisation (Elmfeldt et al., 1975a). The register includes 90 per cent of all surviving diagnosed cases of myocardial infarction in the city. Patients discharged from hospital alive are systematically followed at a special clinic-The Post-MI 
Clinic (Wilhelmsen, 1969; Elmfeldt et al., 1975b). During the years 1968 to 1970,160 men aged 45 to 54 years (mean age 50.3 years) had survived a primary myocardial infarct. Data from these men have been reported earlier (Elmfeldt et al., 1976a, b). Because of the lower incidence of myocardial infarction in women we collected female cases for a longer period from 1968 to 1974. A total of 60 women surviving a primary myocardial infarct in the age group 45 to 54 years (mean age 50.6 years) were registered during this time.

It has been shown that serum cholesterol and blood pressure as well as smoking habits change with myocardial infarction, but studies of a population sample examined before as well as after myocardial infarct have enabled us to allow for the effect of these changes (unpublished observations). Thus, an estimate of the probability of suffering a myocardial infarct has been made retrospectively for the patients-both men and women-in the present study. This was done by means of a multiple risk function developed from a multivariate logistic analysis by Wilhelmsen et al. (1973) modified through an isotonic regression technique (Barlow et al., 1972) adopted by us for computer analysis. This function gives the estimated probability of a 50-year-old man suffering a myocardial infarct during 10 years of follow-up based on three factors, serum cholesterol, systolic blood pressure, and tobacco smoking. The estimated probabilty will be referred to below as 'the risk'. For ease in practical use a nomogram of the risk function was constructed (Fig. 1).

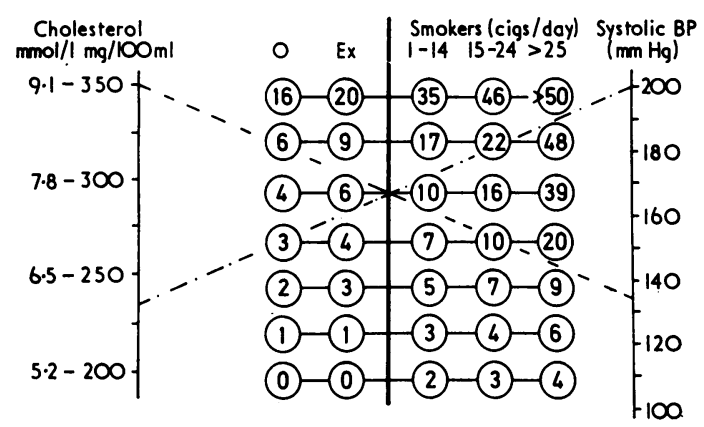

Fig. 1 Nomogram for determination of estimated probability of a 50-year-old man without clinical signs of coronary heart disease suffering myocardial infarction before the age of 60 years. The numbers within circles denote the number of men aged 50 out of 100 who develop myocardial infarction during 10 years' follow-up. By connecting the cholesterol value and systolic blood pressure value of the subject, the vertical line will be crossed near a horizontal line; the risk for the appropriate smoking habit can be found in the circle in the relevant column.
Patients were asked about one week after the myocardial infarct about their smoking habits. According to earlier reports, serum cholesterol had usually returned to preinfarction levels about 3 months after infarction (Tibblin and Cramér, 1963; Cotton, 1970; Fyfe et al., 1971). As we had found the same results in patients controlled by us we used the values from the check-up 3 months after the myocardial infarct (unpublished observations). Blood pressure has been shown to fall during an infarct and the reduction of systolic blood pressure was estimated to be $15 \mathrm{mmHg}$ on average in a special study in which blood pressure values were available before as well as after the myocardial infarct. The distribution of blood pressure decrease was approximately symmetrical, and the decrease was not related to the initial blood pressure level (Wilhelmsen et al., to be published). The values after infarction were, therefore, increased by this amount when calculating the risk.

Of the 160 male patients and 60 female patients discharged alive from hospital, 154 and 53, respectively, were alive 3 months after the acute infarct. Complete data were available for 150 men and 52 women and the risk of these patients was calculated in accordance with the risk function.

For comparison between the male myocardial infarct patients and the general male population, we used data for 50-year-old men from the study of men born in 1913 (Tibblin, 1965, 1967). The participation rate in that study was 88 per cent. Of the 855 men who were examined at entry in 1963, 834 were free of clinical myocardial infarct. These men have been followed for 10 years.

Data from a random population sample of 50year-old women also living in Göteborg were obtained from the study by Bengtsson (1973). From 398 participants all free of clinical myocardial infarct at the first examination in 1968 data for this analysis were available for 396 women. The participation rate in the study of women was 91 per cent. These women bave been followed up for 7 years.

Data from another population sample comprising 387 women aged 52 years in 1974 and examined with the same methods were available for analysis of any time trend between 1968 and 1974 in smoking habits, blood pressure, or serum cholesterol.

Both the patients and the population samples were drawn from the population of the same city and were investigated and interviewed according to standardised methods used during the entire period, by the same team. The methods have been described in detail previously and careful checks of their comparability have been performed (Bengtsson, 1973; Elmfeldt et al., 1975a, b). 
Table 1 Blood pressure, serum cholesterol, serum triglycerides, and smoking habits in men, and pre- and postmenopausal women in the population samples and in those with myocardial infarction

\begin{tabular}{|c|c|c|c|c|c|c|c|c|}
\hline & \multicolumn{4}{|c|}{ Population samples age 50 years } & \multirow{2}{*}{\multicolumn{4}{|c|}{$\begin{array}{l}\text { Myocardial infarct patients from MI-clinic } \\
\text { age } 45 \text { to } 54 \text { years } \\
\text { Men Women 1968-74 }\end{array}$}} \\
\hline & \multirow[t]{2}{*}{ Men } & \multicolumn{3}{|c|}{ Women } & & & & \\
\hline & & Total & Premenopause & Postmenopause & & \multicolumn{2}{|r|}{ Premenopause } & Postmenopause \\
\hline Number & 855 & 384 & 185 & 148 & 150 & 52 & 21 & 31 \\
\hline $\begin{array}{l}\text { Mean } \\
\text { Messure (mming) }\end{array}$ & 138 & 138 & 140 & 135 & 139 & 148 & 148 & 148 \\
\hline SD & 21 & 21 & 22 & 21 & 23 & 22 & 21 & 23 \\
\hline \multicolumn{9}{|l|}{ Serum cholesterol $(\mathrm{mmol} / \mathrm{l})$} \\
\hline Mean & $6 \cdot 4$ & 6.9 & 6.6 & $7 \cdot 2$ & $7 \cdot 2$ & $7 \cdot 1$ & $6 \cdot 7$ & $7 \cdot 3$ \\
\hline \multirow{2}{*}{\multicolumn{9}{|c|}{ Triglycerides (mmol/l) }} \\
\hline & & & & & & & & \\
\hline Mean & $1 \cdot 3$ & 1.0 & 0.9 & $1 \cdot 1$ & 1.8 & 1.8 & $1 \cdot 8$ & 1.8 \\
\hline SD & 0.8 & $0 \cdot 6$ & 0.5 & 0.7 & $1 \cdot 0$ & 0.9 & 0.8 & 0.9 \\
\hline Never smoked $(\%)$ & 24 & 56 & 66 & 47 & 4 & 23 & 15 & 28 \\
\hline Ex-smoker $(\%)$ & 20 & 7 & 7 & 7 & 8 & 7 & 5 & 8 \\
\hline \multicolumn{9}{|l|}{ Smokers (\%) } \\
\hline $1-14 \mathrm{~g} /$ day & 35 & 30 & 23 & 36 & 31 & 29 & 15 & 36 \\
\hline$\geq 15 \mathrm{~g} /$ day & 21 & 7 & 4 & 10 & 57 & 41 & 65 & 28 \\
\hline
\end{tabular}

\section{Results}

Table 1 gives data for blood pressure, serum cholesterol, serum triglycerides, and smoking habits in men and women from the population samples and patients with myocardial infarction.

Table 2 gives a comparison between the two female population samples (age 50 and 52 years, respectively) examined in 1968 and 1974. Lower values for systolic blood pressure $(P<0.01)$, and higher values for cholesterol $(P<0.05)$ and triglycerides $(P<0.01)$ were found among the 52-yearold women, who also smoked more $(P<0.01)$.

The data for women in Table 1 are given for premenopausal and postmenopausal women, respectively. It is to be noted that the blood pressure values were not adjusted (increased by $15 \mathrm{mmHg}$ ) for the myocardial infarction patients in this table.

Table 2 Values from the two female population samples

\begin{tabular}{lcc}
\hline & $\begin{array}{c}1968 \\
50 \text { years } \\
(n=384)\end{array}$ & $\begin{array}{c}1974 \\
52 \text { years } \\
(n=387)\end{array}$ \\
\hline Systolic blood pressure (mmHg) & & \\
$\quad$ Mean & 138 & 133 \\
SD & 21 & 20 \\
Serum cholesterol (mmol/1) & $6 \cdot 9$ & $7 \cdot 1$ \\
$\quad$ Mean & $1 \cdot 1$ & $1 \cdot 2$ \\
SD & $1 \cdot 0$ & $1 \cdot 3$ \\
Triglycerides (mmol/1) & $0 \cdot 6$ & 0.5 \\
$\quad$ Mean & 56 & 50 \\
SD & 7 & 10 \\
Never smoked (\%) & 30 & 24 \\
Ex-smoker (\%) & 7 & 16 \\
Smokers (\%) & & \\
1-14 g/day & & \\
$\geq 15$ g/day & & \\
\hline & &
\end{tabular}

The blood pressure values were higher for both preand postmenopausal women with infarction compared with women from' the general population $(P<0.01)$. Premenopausal women in the population sample had higher blood pressures than postmenopausal women $(P<0.05)$. Men with infarction had similar blood pressure values to men from the general population $(P>0.05)$, but it should be borne in mind that the myocardial infarct had decreased the mean value by about $15 \mathrm{mmHg}$ (after correction $P<0.01$ ).

There were small differences between women with infarction and women from the general population with respect to cholesterol values $(P>0 \cdot 10)$. For the population sample of women a pronounced difference in mean values was found for women before and after the menopause $(P<0.01)$, and a similar but non-significant difference was found for the women with infarction $(P>0.05)$. However, there was still no difference between women with infarction and the general population when allowance was made for the effect of menopause. For men the expected difference in cholesterol values between men with infarction and men in the general population was found.

There was a difference in triglyceride values between women with infarction and women from the general population $(P<0.01)$. The triglyceride values were higher in the postmenopausal than in the premenopausal women in the general population $(P<0.01)$, but for the women with infarction no such tendency was found. Thus, the difference was somewhat greater in the premenopausal than in the postmenopausal women. The men with infarction also had higher triglycerides than the men from the general population $(P<0.01)$. 
The correlation coefficients between cholesterol and triglycerides were 0.33 to 0.38 for both men and women in the population samples as well as in the infarct groups. There were no differences in correlation coefficients if the women were divided with respect to menopause.

The women with infarction were much more often smokers than women in the general population $(P<0.01)$. An especially pronounced difference compared with the general population was found for premenopausal women with infarction among whom 65 per cent smoked 15 cigarettes a day or more as compared with 4 per cent in the general population. The postmenopausal women in the general population smoked more $(P<0.01)$ than the premenopausal women.

If the women with infarction are compared with the population sample of women examined in 1974 (aged 52 years) there is still a difference in systolic blood pressure $(P<0.01)$, no difference in cholesterol values, and still a pronounced difference in triglycerides $(P<0.01)$. Even though the women examined in 1974 smoked more than those examined in 1968, there is still a pronounced difference $(\mathrm{P}<0.01)$.

Fig. 2 shows the distribution of risk of myocardial infarct according to the multiple risk function for the random population sample of men and for the 150 men with primary myocardial infarct, as well as the same distributions for women in the female population sample and the 52 women with primary myocardial infarct (blood pressure values now adjusted by $15 \mathrm{mmHg}$ ). The distributions of risk in the male and female population samples were similar. Thus, 89 per cent of both men and women had a predicted risk of 0.10 or less, and 2.6 per cent of the men and 2.5 per cent of the women had a risk of 0.20 or higher.

When comparing men and women with myocardial infarction it appears that the distributions with respect to risk also are very similar (Fig. 2); 35 per cent of the men and 40 per cent of the women with myocardial infarct had a predicted risk of 0.10 or less.

The number of men and women in the population samples who suffered a myocardial infarct during 10 years' and 7 years' follow-up, respectively, is shown in Table 3. The numbers are given in relation to classes of multiple risk. As the prediction formula was based on the incidence of myocardial infarction in the sample of men, it is self evident that the incidence must increase considerably with increasing values for estimated risk among the men, but there are evidently some men who suffered a myocardial infarct in spite of low risk as judged by cholesterol values, blood pressure values, and smoking habits. Of the 26 non-fatal myocardial infarct cases 18 $(69 \%)$ and of the 18 fatal cases $9(50 \%)$ had an estimated risk of 0.10 or less. Not a single woman in the population sample suffered a myocardial infarct during the 7 years' follow-up.

As seen in Table 4, 21 of the 52 women with myocardial infarction (all aged 45 to 54 years) were premenopausal. The mean ages of the premenopausal and postmenopausal women were 48.4 and 51.9 years, respectively. (Of the 31 postmenopausal

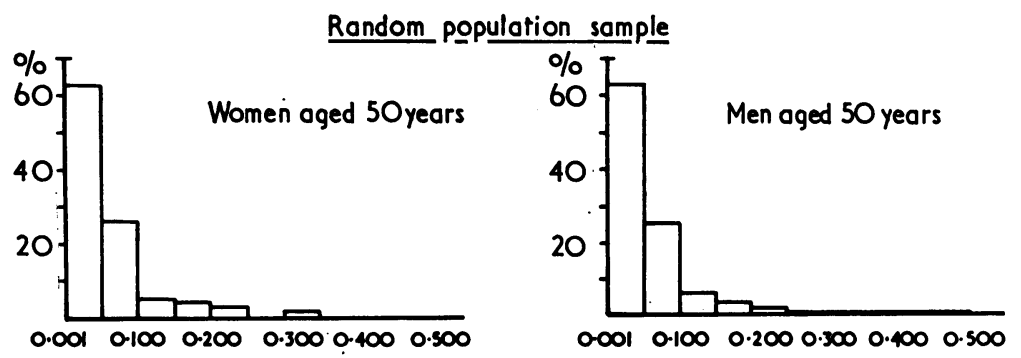

Fig. 2 Estimated probability of suffering a myocardial infarct according to a multiple logistic function in a random sample of men aged 50 years (top right), men (45-54 years. old) who have survived a myocardial infarct (bottom right) compared with a random sample of women aged 50 years (top left) and women (45-54 years) who have survived

Myocardial inforction survivors

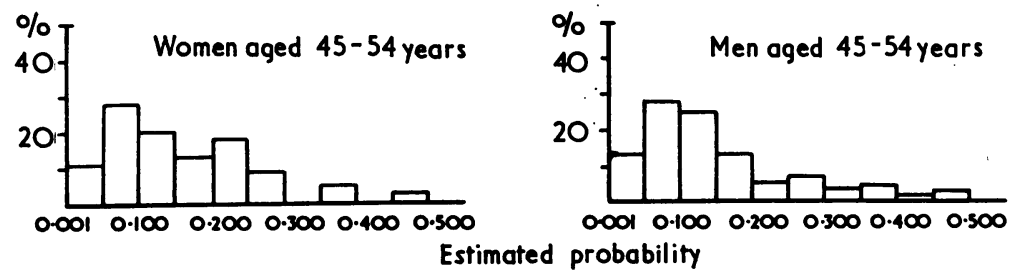
a myocardial infarct (bottom left). 
women, 3 had their menopause prematurely as a result of operations.) The median risk in premenopausal women was 0.13 and in the postmenopausal women $0 \cdot 11$. Of the 52 myocardial infarct women 16 were between 45 and 49 years; 11 of these were premenopausal and their median risk was the same as for the postmenopausal women in this age group. Among postmenopausal women there was no tendency towards a shorter time interval between menopause and acute myocardial infarction in women with the highest risk.

As mentioned above, cholesterol was not found to be associated with myocardial infarction in the women. Triglycerides were, however, higher in women with myocardial infarction. The possibility that women with infarction and low multiple risk had higher triglycerides than women with infarction and high multiple risk was examined but this was not found to be so. There was a pronounced increase in triglycerides with increasing multiple risk, and this is because of positive bivariate correlations between triglycerides and cholesterol $(r=0.38)$, blood pressure $(r=0.10)$, and smoking $(r=0.16)$ in the women with infarction.

Six women had diabetes mellitus detected by glycosuria and 5 of these were between 50 and 54 years. The median risk in these 6 women was 0.10.

\section{Discussion}

It is difficult to assess the importance of risk factors which occur infrequently in the population, just as it is difficult to come to any conclusions concerning risk factors if the incidence of a disease is low. The former is a problem when, for example, diabetes is studied. In several prospective population studies diabetes has not been detected as a significant risk factor because manifest diabetes has a prevalence of only around 1 per cent in middle-aged men and women. By comparing patients who have already suffered a myocardial infarct with the general population it has, however, been possible to show the importance of diabetes as a risk factor in women and men in the Swedish population (Bengtsson, 1973; Elmfeldt et al., 1976a, c). It has not been possible to assess the risk factors in the prospective population study of women during 7 years' followup since there have as yet been no episodes of myocardial infarction.

The methods used in this study have made it possible to examine the risk characteristics of Swedish women with myocardial infarction. It must, however, be stressed that a certain bias might have been introduced as the study only included non-fatal cases of myocardial infarction. That there

Table 3 Number of men and women suffering a myocardial infarct (MI) in relation to multiple risk prediction

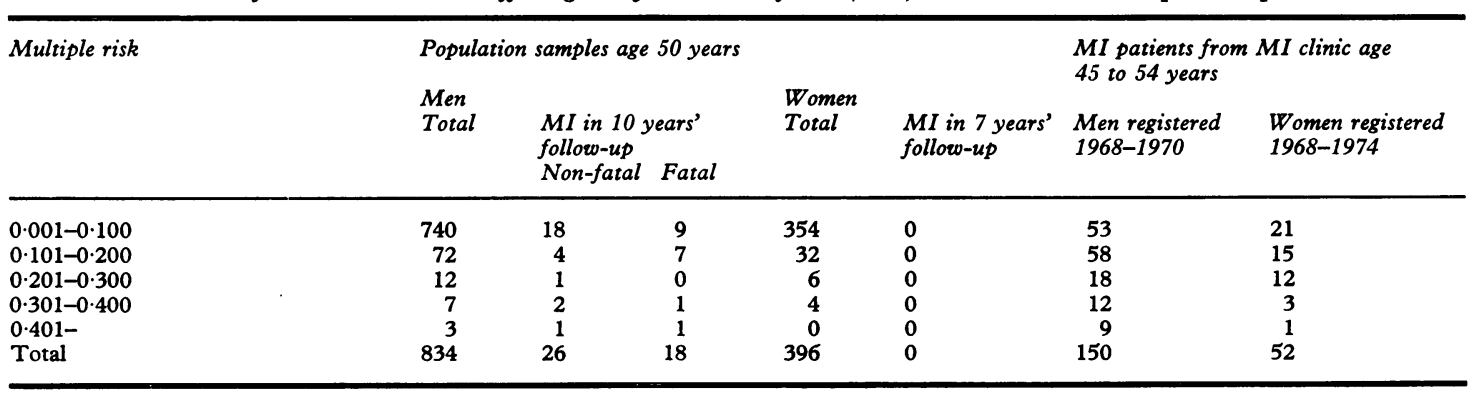

Table 4 Multiple risk, median age, and triglycerides for pre-and postmenopausal women with myocardial infarction (MI)

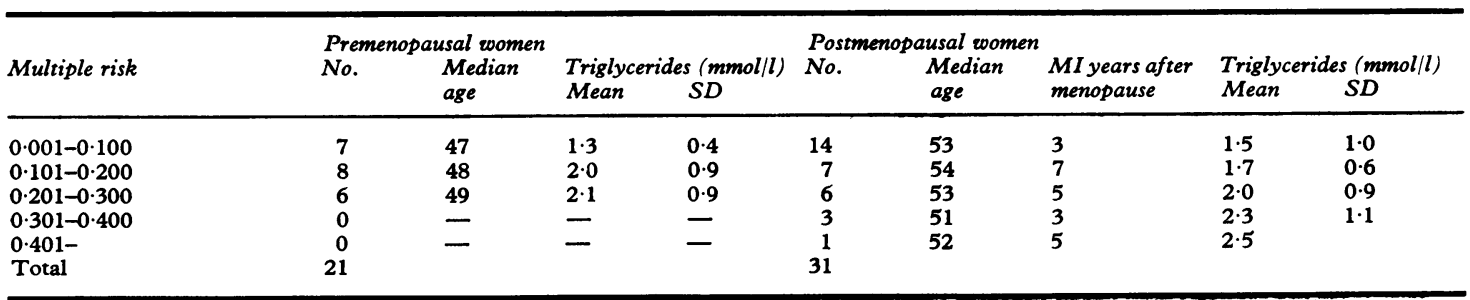


may be differences in risk factor profiles between non-fatal and fatal myocardial infarction sufferers has been discussed in previous papers (Tibblin et al., 1975; Elmfeldt et al., 1976a, b, c). In a previous analysis it was found that hypertension, high triglycerides, smoking, and diabetes mellitus were more common among the women with myocardial infarction than among the general population (Bengtsson, 1973). The prevalence of diabetes mellitus among the women with myocardial infarction-15 per cent-was higher than the figure for diabetes among men with myocardial infarction -7 per cent. In the population samples diabetes was slightly more prevalent among women than men-1.3 and 0.8 per cent, respectively, but the difference is not statistically significant.

It seems reasonable to assume that at least some of the factors which are related to the differences between the sexes are connected with the precipitation of myocardial infarction and not the atherosclerotic process as such. It is evident from Table 3 and Fig. 2 that the protective effect of female sex is active irrespective of the multiple risk based upon the levels of blood pressure, serum cholesterol, and smoking habits.

The present analysis aimed at testing whether women, who had suffered a myocardial infarct despite the overall low incidence of this disease in middle-aged women, were more heavily burdened by the risk factors hypercholesterolaemia, hypertension, and smoking than are men with myocardial infarction.

We found, however, similar distributions of these risk factors in myocardial infarct patients in the two sexes and in general between pre- and postmenopausal women, which speaks against this hypothesis.

As in the Framingham study, it was found that serum cholesterol was higher in postmenopausal than in premenopausal women (Hjortland et al., 1976). The present results indicate that high serum cholesterol is not such a strong risk factor for (nonfatal) myocardial infarction in women as in men. High triglycerides are apparently more common in infarct women. This finding is puzzling and difficult to explain in view of the fairly strong correlation between cholesterol and triglycerides in women and in men. The present results suggest that a high triglyceride level is an independent risk factor for myocardial infarction in women as opposed to the finding in men (Wilhelmsen et al., 1973), but because of the lack of prospective data in women it cannot be proved by this study.

As only 6 women with myocardial infarct had diabetes, the higher prevalence of diabetes among women than among men with myocardial infarct can only partly explain why the influence of the female sex could not protect these particular women from myocardial infarction. Even though there were also some differences with respect to mental stress, certain personality traits, and physical inactivity between women with myocardial infarcts and the general population of women (Bengtsson, 1973), we cannot see that these differences can 'explain' why the women suffered myocardial infarction. Thus, we conclude from the present study that some other factor or factors must be responsible.

It is evident that there are as yet unidentified factors which are important for premature myocardial infarction. In this context it is interesting to compare the present findings with those of Keys (1972) who found that there must be some unknown risk factor or factors which explain the higher rate of myocardial infarction among American as compared with European men. We can only hypothesise about the possible mechanisms of such factors. Thus, they may have to do with the lipid metabolism of the vessel wall, the coagulation system, or even with the sensitivity of the myocardium to hypoxia or its tendency to react with catecholamine release. Wolinsky (1973) has shown experimentally that the vessel wall may react differently in male and female animals. If there are similar sex differences (brought about by oestrogens or androgens) with respect to the sensitivity of the myocardium in humans, they are not known at present, but are worthy of investigation.

Supported by grants from the Swedish National Organization against Heart and Chest Diseases.

\section{References}

Barlow, R. E., Bartholomew, D. J., Bremner, J. M., and Brunk, H. D. (1972). Statistical Inference Under Order Restrictions. John Wiley, New York.

Bengtsson, C. (1969). The women study 1968-1969. Pehr Dubb fournal (Göteborg), 4, 4, 14-19.

Bengtsson, C. (1973). Ischaemic heart disease in women. Acta Medica Scandinavica, Suppl. 549.

Cotton, S. G. (1970). Plasma cholesterol after myocardial infarction. Postgraduate Medical fournal, 46, 551-552.

Elmfeldt, D., Vedin, J. A., Wilhelmsson, C., Tibblin, G., and Wilhelmsen, L. (1976a). Morbidity in representative male survivors of myocardial infarction compared to representative population samples. Fournal of Chronic Diseases, 29, 221-231.

Elmfeldt, D., Wilhelmsen, L., Tibblin, G., Vedin, J. A., Wilhelmsson, C., and Bengtsson, C. (1975a). Registration of myocardial infarction in the city of Göteborg, Sweden. Fournal of Chronic Diseases, 28, 173-186.

Elmfeldt, D., Wilhelmsen, L., Tibblin, G., Vedin, J. A., Wilhelmsson, C., and Bengtsson, C. (1975b). A postmyocardial infarction clinic in Göteborg, Sweden. Acta Medica Scandinavica, 197, 497-502.

Elmfeldt, D., Wilhelmsson, C., Vedin, J. A., Tibblin, G., and Wilhelmsen, L. (1976b). Characteristics of representative 
male survivors of myocardial infarction compared with representative population samples. Acta Medica Scandinavica, 199, 387-398.

Elmfeldt, D., Wilhelmsen, L., Wedel, H., Vedin, A., Wilhelmsson, C., and Tibblin, G. (1976c). Primary risk factors in patients with myocardial infarction. American Heart fournal, 91, 412-419.

Fyfe, T., Baxter, R. H., Cochran, K. M., and Booth, E. M. (1971). Plasma-lipid changes after myocardial infarction. Lancet, 2, 997-1001.

Hjortland, M. C., McNamara, P. M., and Kannel, W. B. (1976). Some atherogenic concomitants of menopause: the Framingham study. American fournal of Epidemiology, 103, 304-311.

Kannel, W. B., and Castelli, W. P. (1972). The Framingham study of coronary disease in women. Medical Times (New York), 100, 173-195.

Kannel, W. B., Hjortland, M., and McNamara, P. (1975). Menopause and risk of cardiovascular disease: the Framingham study. Abstract of Conference on CVD Epidemiology, Tampa, Florida, 10-11 March.

Keys, A. (1972). Predicting coronary heart disease. In Preventive Cardiology, pp. 21-31. Ed. by G. Tibblin, A. Keys, and L. Werkö. John Wiley, New York.

Tibblin, G. (1965). A population study of 50-year-old men. An analysis of the non-participation group. Acta Medica Scandinavica, 178, 453-459.
Tibblin, G. (1967). High blood pressure in men aged 50-a population study of men born in 1913. Acta Medica Scandinavica, Suppl. 470.

Tibblin, G., and Cramer, K. (1963). Serum lipids during the course of an acute myocardial infarction and one year afterwards. Acta Medica Scandinavica, 174, 451-455.

Tibblin, G., Wilhelmsen, L., and Werkö, L. (1975). Risk factors for myocardial infarction and death due to ischemic heart disease and other causes. American fournal of Cardio$\log y, 35,514-522$.

Wilhelmsen, L. (1969). The myocardial infarction clinic in Göteborg-organization and preliminary results. Pehr Dubb fournal, 4, 43-54.

Wilhelmsen, L., Wedel, H., and Tibblin, G. (1973). Multivariate analysis of risk factors for coronary heart disease. Circulation, 48, 950-958.

Wolinsky, H. (1973). Comparative effects of castration and antiandrogen treatment on the aortas of hypertensive and normotensive male rats. Circulation Research, 33, 183-189.

Requests for reprints to Dr. L. Wilhelmsen, Department of Medicine I, Sahlgrenska Hospital, S-413 45 Göteborg, Sweden. 